\title{
Case 1 - Successful resection after FOLFIRI plus cetuximab therapy for unresectable colorectal cancer with multiple liver metastases
}

\author{
Anna Nappi ${ }^{1}$, Carmela Romano ${ }^{1}$, Gugliemo Nasti ${ }^{1}$ \\ Commentary: Fotios Loupakis
}

\begin{abstract}
This case report describes the outcome of a multidisciplinary approach which allowed successful resection in a patient diagnosed with unresectable colorectal cancer. The patient was a 66-year-old man with no important comorbidities. His main complaint was abdominal pain. The patient received a diagnosis of moderate differentiated sigmoid colon adenocarcinoma, $R A S$ and $B R A F$ wild type. Multiple liver metastases were detected. Conversion therapy was given and the patient received 8 therapy courses with FOLFIRI plus cetuximab. Grade 1 diarrhea and grade II skin toxicity were observed as adverse effects of the chemotherapy. The size of the tumor was reduced and the tumor was resected performing left colectomy, segment liver VI resection and performing intraoperative thermos-ablation with microwave. No recurrence was observed at 6 months after surgery.
\end{abstract}

Key words: left colorectal cancer, liver metastasis, multidisciplinary approach, conversion chemotherapy

\section{Introduction}

In this paper, we present and discuss the case of a patient affected by left colorectal cancer (CRC). At the time of diagnosis, the patient presented unresectable disease due to the presence of multiple liver metastases. This case points out the importance of personalized therapy based on the molecular characteristics of disease and emphasizes the importance of a multidisciplinary approach to clinical decision making in the treatment of metastatic disease.

\section{Case description}

A 66 year-old man on December 2015 presented constipation and frequent abdominal pain. The family doctor recommended analysis to detect the presence of occult blood in the stool, which was confirmed by the analysis. There-

\footnotetext{
'Department of Abdominal Oncology, Istituto Nazionale Tumori di Napoli "G. Pascale" IRCCS, National Cancer Institute, Naples, Italy. Correspondence to:

Anna Nappi, MD, PhD student

Dipartimento di Oncologia Addominale,

Istituto Nazionale Tumori IRCCS Fondazione G. Pascale,

via M. Semmola 52, 80131 Napoli, Italy.

Phone: +390815903367 - Fax: +390815903822

E-mail: anna2184@hotmail.it

CANCER BREAKING NEWS 2017;5(3):30-35

DOI: $10.19156 / c b n .2017 .0057$
}

fore, the patient underwent a colonoscopy that showed the presence of a suspected mass at the sigmoid colon. This mass showed a reduction in the intestinal lumen, and was bleeding. During colonoscopy, this mass was subjected to biopsy, and the subsequent histological examination showed the presence of a moderately differentiated adenocarcinoma [1]. At this time the patient was referred to our center, the National Cancer Institute of Naples, Italy. The patient's medical history was unremarkable and consisted only of well-controlled hypertension. He reported suffering from constipation and abdominal pain, symptoms both of which had deteriorated in recent months.

Clinical examination showed a small increase in liver size and abdominal palpation was painful.

Baseline computed tomography (CT) scan showed the presence of a mass at the sigmoid colon of about $6 \mathrm{~cm}$ and multiple liver metastases, the larger ones were localized at segment IVa $(55 \mathrm{~cm})$ and at segment VI $(30 \mathrm{~cm})$ (Figure 1).

The patient's baseline carcinoembryonic antigen (CEA) value was $743 \mathrm{ng} / \mathrm{mL}$. To confirm the diagnosis, the patient underwent a positron emission tomography (PET) scan that showed a high fluorodeoxyglucose (FDG) avidity of lesions with SUV 9 (sigmoid colon) and SUV 11 (liver), without other pathological findings. Given the extent of the disease, analysis of $R A S$ and $B R A F$ mu- 


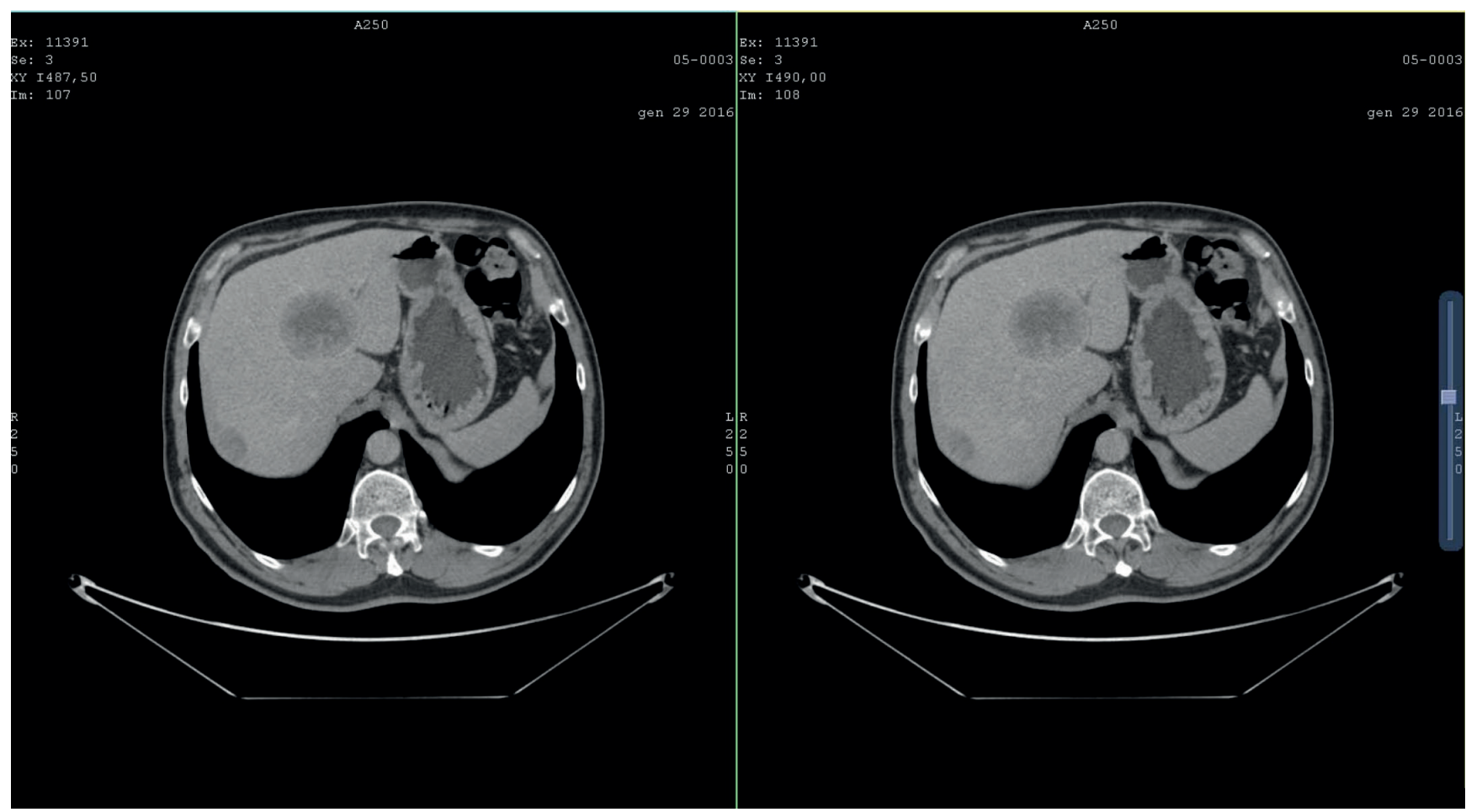

Fig. 1. Baseline CT scan showing multiple liver metastases.

tational status was required. The analysis showed the absence of mutation in all genes analyzed (KRAS, NRAS and $B R A F)$. After fully informing the patient about the procedure and implantation of a port-a-cath, palliative first-line treatment with 5-fluorouracil based chemotherapy plus cetuximab was initiated.

From February 02, 2016, the patient received cetuximab on day 1 of a 8-day treatment cycle, (initial dose 400 $\mathrm{mg} / \mathrm{m}^{2}$ of body surface area infused over $120 \mathrm{~min}$, thereafter $250 \mathrm{mg} / \mathrm{m}^{2}$ of body surface area infused over 60 min weekly) plus FOLFIRI (irinotecan, 5-fluorouracil and leucovorin) on day 1 of a 14-day treatment cycle, (comprising a 60- to 90-min infusion of irinotecan at a dose of $180 \mathrm{mg} / \mathrm{m}^{2}$ of body surface area, a 120-min infusion of racemic folinic acid at a dose of $400 \mathrm{mg} / \mathrm{m}^{2}$ of body surface area, and 5-fluorouracil as an i.v. bolus of $400 \mathrm{mg} / \mathrm{m}^{2}$ of body surface area, and then a continuous 46 -hour infusion of $2,400 \mathrm{mg} / \mathrm{m}^{2}$ of body surface area). Treatment was well tolerated, apart from Common Toxicity Criteria (CTC) grade I diarrhea and skin toxicity consisting of CTC grade II acneiform rash [2]. After four fortnightly cycles of FOLFIRI plus cetuximab (2 months), a follow-up CT scan showed a large reduction in size of all target lesions (Figure 2). The overall response was a partial response.

In agreement with the will of the patient, we decided to continue the treatment for four cycles.

After four months of treatment, follow-up PET was negative, and the global response was complete metabolic response. The patient then continued treatment with cetuximab as maintenance therapy until December 2016 when a CT scan showed stable disease and his CEA value was $6.1 \mathrm{ng} / \mathrm{mL}$. Following presentation of the case to the multidisciplinary tumor board, surgical resection was recommended.

On February 14, 2017, following laparotomy, left colon, gallstones and liver segments VI were resected, and intraoperative thermo-ablation with microwave of segments IV was performed. Histological examination showed the presence of sigmoid colon adenocarcinoma (stage pT2 N1 V0) [3], and the presence of fibrosis in liver segment VI; all resected had negative margins. Postsurgical healing was without complications. The patient returned to normal everyday life within one month. He is now fully active with no sign of disease relapse detected at CT scan evaluation performed 2 and 4 months after surgery.

\section{Discussion}

Colorectal cancer is the second most commonly diagnosed cancer in Europe and a leading cause of death both in Europe and worldwide. About 20\% of patients with CRC present metastasis at diagnosis, and about $35 \%$ of patients treated with healing intent will develop metastases [4]. Over the last decade, the clinical outcome for patients with metastatic CRC (mCRC) has improved, due to changes in 


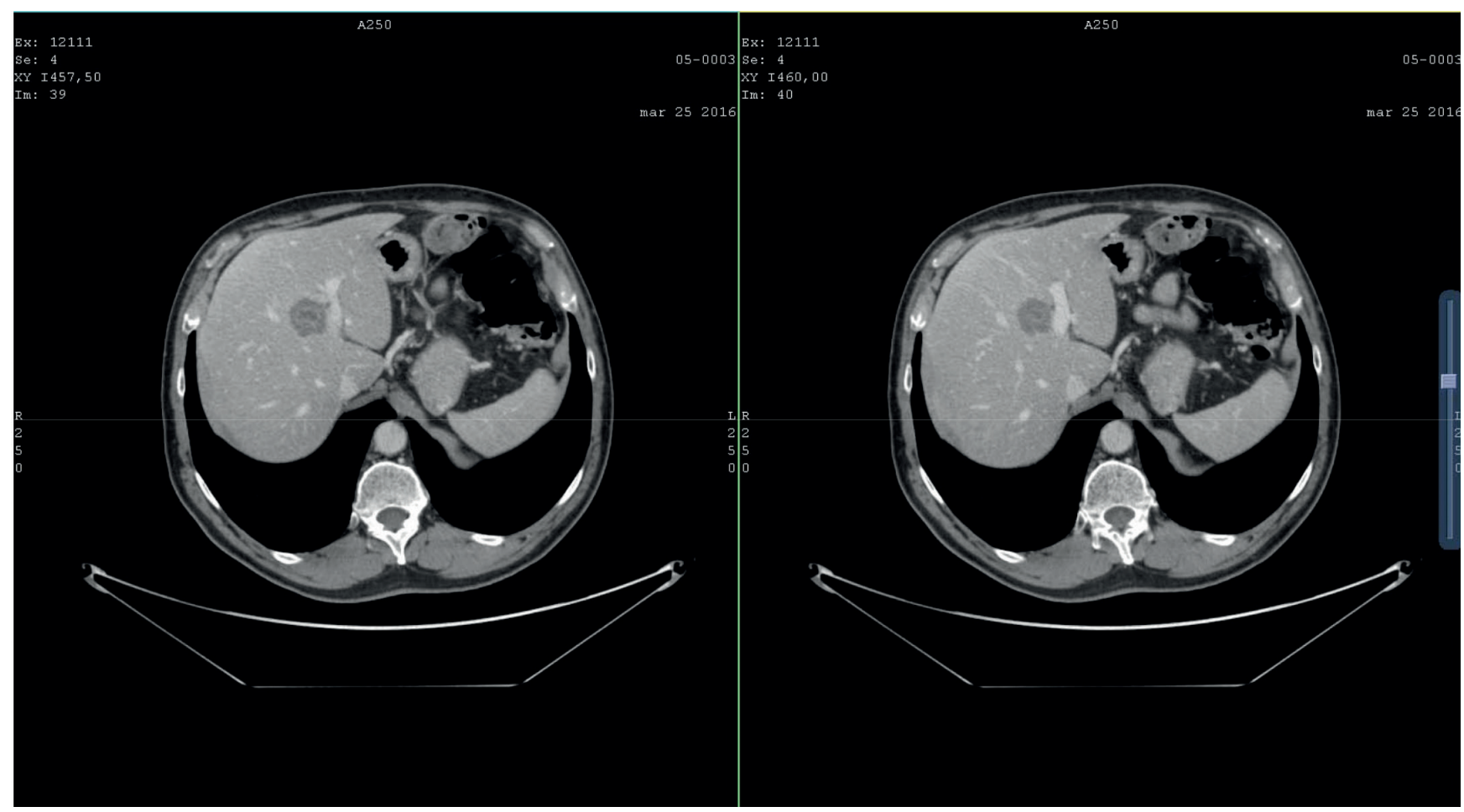

Fig. 2. CT scan after 4 cycles of treatment showing a reduction in size of all liver metastases.

the clinical presentation of patients, improvements in the efficacy of systemic therapies and implementation of 'continuum of care' treatment strategies. Molecular characteristics play a crucial role in the treatment of metastatic disease. Recent evidence from the PRIME study with panitumumab [5], from the CRYSTAL study with cetuximab [6] and from other studies of epidermal growth factor receptor (EGFR) monoclonal antibody therapies has shown that mutations in the KRAS exon 2, 3 and 4 and in NRAS exon 2, 3 and 4 (expanded $R A S$ analysis) predict a lack of response to EGFR-targeting monoclonal antibodies, and that these therapies may in fact have a detrimental effect in patients with $R A S$ mutant disease [7].

In the phase III CRYSTAL study, which randomized patients to receive first-line FOLFIRI with or without cetuximab, other $R A S$ mutations were detected in nearly $15 \%$ of evaluable patients previously assessed to be $K R A S$ exon 2 wild-type. In patients with $R A S$ wild-type tumors, the addition of cetuximab to FOLFIRI was associated with improved treatment outcomes across all efficacy end points. Conversely, in patients with $R A S$ mutant tumors, no benefit from the addition of cetuximab to FOLFIRI versus FOLFIRI alone was observed [6].

Data from the phase III FIRE-3 trial also underscore the importance of expanded $R A S$ mutational analysis. The authors observed an improvement in overall survival (OS) for patients with $R A S$ wild-type tumors treated with cetuximab [8]. The cumulative data clearly show that patients whose tumors harbor any $R A S$ mutation are unlikely to benefit from EGFR antibody therapy, confirming the presence of a $R A S$ mutation as a negative predictive marker of treatment outcome in patients with mCRC.

For patients with liver limited disease, the aim of treatment is complete resection. Studies involving the retrospective analysis of response rate (RR) in patients with liver limited disease and the corresponding compete resection rates provide additional information $[9,10]$, but need to be regarded with caution. However, it seems clear that regimens that achieve high RRs are beneficial and are associated with higher complete resection rates. Thus, the standard chemotherapy regimens used in the CRYSTAL [6], PRIME [10] and OPUS [11] trials with EGFR-targeting monoclonal antibodies versus chemotherapy alone in patients with $R A S$ wild-type disease should be regarded as standard treatment options. Moreover, data from the FIRE-3 [8] and CALGB [12] studies show that a cytotoxic doublet plus cetuximab in $R A S$ wild-type patients is associated with higher RRs compared with bevacizumab, although this did not translate into higher resection rates in either of these studies.

The literature data show that patients whose primary tumours originate on the left side of the colon (the descending colon, sigmoid colon, and rectum) survive 
significantly longer than those whose tumours originate on the right side (the cecum and ascending colon). In a recent retrospective analysis, the authors investigated the prognostic and predictive influence of the localization of the primary tumor in patients with unresectable $R A S$ wild type mCRC included in six randomized trials (CRYSTAL, FIRE-3, CALGB 80405, PRIME, PEAK and 20050181), comparing chemotherapy plus EGFR antibody therapy with chemotherapy or chemotherapy plus bevacizumab. Primary tumor location and $R A S$ mutation status were available for $37.5 \%(2159 / 5760)$ of patients randomized across the 6 trials (515 right-sided and 1644 left-sided). The authors observed a significantly worse prognosis for patients with right-sided tumours compared with those with left-sided tumours for OS, progression free survival (PFS) and objective response rate (ORR). In terms of a predictive effect, a significant benefit for chemotherapy plus EGFR antibody therapy was observed in patients with left-sided tumours compared with no significant benefit for those with rightsided tumours. For ORR, there was a trend towards a greater benefit for chemotherapy plus EGFR antibody therapy in the patients with left-sided tumours compared with those with right-sided tumours [13].

\section{Conclusions}

The optimal treatment strategies for patients with $\mathrm{mCRC}$ are evolving rapidly with improved clinical outcomes being achieved when the treatment approaches

\section{References}

1. Guinney J, Dienstmann R, Wang X et al. The consensus molecular subtypes of colorectal cancer. Nat Med 2015;21(11):1350-6.

2. National Institutes of Health/National Cancer Institute. Common Terminology Criteria for Adverse Events (CTCAE) version 4.0. 2009.

3. Sobin LH, Gospodarowicz MK, Wittekind C. TNM classification of malignant tumours 7 th edition. Wiley-Blackwell; 2009.

4. Ferlay J, Soerjomataram I, Dikshit R et al. Cancer incidence and mortality worldwide: sources, methods and major patterns in GLOBOCAN 2012. Int J Cancer 2015;136(5):E359-86.

5. Douillard JY, Oliner KS, Siena S et al. PanitumumabFOLFOX4 treatment and RAS mutations in colorectal cancer. N Engl J Med 2013;369(11):1023-34.

6. Van Cutsem E, Lenz HJ, Kohne CH et al. Fluorouracil, leucovorin, and irinotecan plus cetuximab treatment and RAS mutations in colorectal cancer. J Clin Oncol 2015;33(7):692-700

7. Stintzing S, Miller-Phillips L, Modest DP et al. Impact of BRAF and RAS mutations on first-line efficacy of FOLFIRI plus cetuximab versus FOLFIRI plus bevacizumab: for individual patients are discussed within a multidisciplinary tumor board of experts who meet regularly to review metastatic colorectal cases [14]. The role of the multidisciplinary tumor board is to define the initial diagnostic workup and then the treatment focus, based on the best diagnostic and therapeutic decision-making available, such as in our case. Furthermore, a multidisciplinary team-managed treatment strategy has to be maintained for the duration of a patient's treatment, to allow the refinement of treatment strategies according to on-treatment information and evaluation of the potential need for the integration of ablative treatments. Patients suffering from mCRC may benefit from a multidisciplinary approach; this approach can be successfully applied following a response to palliative first-line treatment, and a curative option for these patients may be possible. Regular presentation of imaging findings to an interdisciplinary tumor board highly experienced in surgical oncology is important for enabling the prognosis of these patients.

\section{Acknowledgments}

The Authors thank Ray Hill, an independent medical writer, who provided native English editing and journal styling on behalf of HPS.

\section{Conflicts of Interest}

The Authors declare there are no conflicts of interest in relation to this article.

analysis of the FIRE-3 (AIO KRK-0306) study. Eur J Cancer 2017;79:50-60.

8. Heinemann V, von Weikersthal LF, Decker T et al. FOLFIRI plus cetuximab versus FOLFIRI plus bevacizumab as first-line treatment for patients with metastatic colorectal cancer (FIRE-3): a randomised, open-label, phase 3 trial. Lancet Oncol 2014;15(10):1065-75.

9. Van Cutsem E, Kohne CH, Lang I et al. Cetuximab plus irinotecan, fluorouracil, and leucovorin as first-line treatment for metastatic colorectal cancer: updated analysis of overall survival according to tumor KRAS and BRAF mutation status. J Clin Oncol 2011;29(15):2011-9.

10. Douillard JY, Siena S, Cassidy J et al. Final results from PRIME: randomized phase III study of panitumumab with FOLFOX4 for first-line treatment of metastatic colorectal cancer. Ann Oncol 2014;25(7):1346-55.

11. Bokemeyer C, Bondarenko I, Hartmann JT et al. Efficacy according to biomarker status of cetuximab plus FOLFOX-4 as first-line treatment for metastatic colorectal cancer: the OPUS study. Ann Oncol 2011;22(7):1535-46.

12. Venook AP, Niedzwiecki D, Lenz H-J et al. CALGB/SWOG 
80405: Phase III trial of irinotecan/5-FU/leucovorin (FOLFIRI) or oxaliplatin/5-FU/leucovorin (mFOLFOX6) with bevacizumab (BV) or cetuximab (CET) for patients (pts) with KRAS wild-type (wt) untreated metastatic adenocarcinoma of the colon or rectum (MCRC) [Abstract]. J Clin Oncol 2014;32(15 (Suppl)).

13. Arnold D, Lueza B, Douillard JY et al. Prognostic and predictive value of primary tumour side in patients with RAS wild-type metastatic colorectal cancer treated with chemotherapy and EGFR directed antibodies in six randomised trials. Ann Oncol 2017 Apr 12 [Epub ahead of print].

14. Shah S, Arora S, Atkin G et al. Decision-making in Colorectal Cancer Tumor Board meetings: results of a prospective observational assessment. Surg Endosc 2014; 28(10):2783-8.

\section{Commentary}

Nappi and colleagues report on a patient with synchronous metastatic colorectal cancer (mCRC) with liver-limited disease achieving a response with FOLFIRI plus cetuximab and undergoing local treatment (surgery + microwave ablation) with curative intent.

What's new? I thought back about my colorectal cancer patients in the last 10 years and I remember a strikingly similar case presenting with the same general characteristics (age and disease extension), treated exactly the same way (doublet chemo plus anti-EGFR) in 2007. Even more noticeable, the outcome was very similar with the achievement of a major shrinkage and secondary resection.

Does this mean that we didn't make so much improvement in the treatment of $m C R C$ in 10 years? This feeling of disappointment has driven the mood of many GI oncologists since a while. Nevertheless, the reality is a little bit different.

First of all, 10 years ago we didn't have any real biomarker. The clinical application of codons 12 and 13 KRAS mutations was at its outset [1].

Since then, RAS rare mutations and NRAS testing entered the practice [2]. BRAF mutations, even if still under discussion from a formal point of view, are routinely used by most of the oncologist and now recommended by major guidelines for guiding treatment choice toward approaches different from the anti-EGFR strategy [3, 4]. In line with this, the case presented was RAS and BRAF wild-type and received an anti-EGFR.

The intensity of the chemotherapeutic backbone has been extensively investigated in the last years, clearly establishing different roles for doublets, fluoropyrimidine monotherapy and triplet regimens [4]. In line with this, the choice of the anti-EGFR in the clinical case drove the decision of adopting a doublet chemotherapy as backbone.

Primary tumor location (i.e. right-sided vs left-sided primary colorectal cancer) has been established as a prognostic and predictive factor to bevacizumab vs anti-EGFRs [5]. In line with this, the use of an anti-EGFR was strongly supported by the location of the primary in the sigmoid colon.

The role of the combined approach of surgery and local treatments showed a clear superiority in a recently updated randomized study. In line with this, the patients underwent surgical resection combined to microwave ablation [6].

In other words, what 10 years ago happened by chance and a therapeutic success as the one reported in the clinical case discussed by Nappi et al. was exceptional, nowadays can be achieved much more often as the direct result of a complex decision-making process. It's true that with respect to other oncological diseases the treatment of colorectal cancer did not change too much in terms of pharmacological options, but this apparent lack of progress was largely compensated by the advancements achieved in terms of biomarkers, clinical selection and a better understanding of how to exploit our limited tools. 


\section{References}

1. Lièvre A, Bachet JB, Le Corre D et al. KRAS mutation status is predictive of response to cetuximab therapy in colorectal cancer. Cancer Res 2006 Apr 15;66(8):3992-5.

2. Baselga J, Rosen N. Determinants of RASistance to antiepidermal growth factor receptor agents. J Clin Oncol 2008 Apr 1;26(10):1582-4.

3. Pietrantonio F, Petrelli F, Coinu A et al. Predictive role of BRAF mutations in patients with advanced colorectal cancer receiving cetuximab and panitumumab: a meta-analysis. Eur J Cancer 2015 Mar;51(5):587-94.

4. Van Cutsem E, Cervantes A, Adam R et al. ESMO consensus guidelines for the management of patients with metastatic colorectal cancer. Ann Oncol 2016 Aug;27(8):1386-422.

5. Arnold D, Lueza B, Douillard JY et al. Prognostic and predictive value of primary tumour side in patients with RAS wild-type metastatic colorectal cancer treated with chemotherapy and EGFR directed antibodies in six randomized trials. Ann Oncol 2017 Aug 1;28(8):1713-29.

6. Ruers T, Van Coevorden F, Punt CJ et al. Local treatment of unresectable colorectal liver metastases: results of a randomized phase II trial. J Natl Cancer Inst 2017 Sep $1 ; 109(9)$. 